Historic, Archive Document

Do not assume content reflects current scientific knowledge, policies, or practices. 



\section{Wholesale Price List to the Trade - - for - -}

\section{Spring 1929 Surplus Stock}

L. J.Rambo's Wholesale Nurseries Bridgman, Michigan

\section{: : References :}

Bridgman State Bank, Bridgman, Michigan stevensville State Bank, Sterensville, Mich. Any Nurseryman in Berrien County, Michigan

All the stock we offer for sale is young, thrifty, well-grown, well-graded and first-class in every way.

Certificate of inspection accompanies each shipment.

We Guarantee Our Stock-to be true to name; of quality as represented; packed carefully to insure arrival in good condition. In case any should prove otherwise, we hold ourselves liable only for the purchase price.

TERMS:-Cash with order, or satisfactory references from unknown parties.

All shipments travel at the risk and cost of the purchaser.

For Spring 1929. - We will book on those varieties at prices named, subject to prior sales, as long as the supply lasts.

When cash accompanies shipment, no charge will be made for packing; otherwise, we make a charge to cover expenses of boxes. No charges made for carlots, loaded in bulk.

The articles in this list will be furnished 50 at 100 rate; and 500 at 1000 rate. The quality is excellent, satisfaction guaranteed. 
(Black Varieties)

-1 Yr. No. 1 and 2 Yr. No. 2-

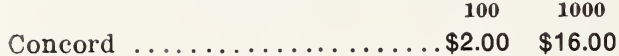

Champion ................. $2.25 \quad 20.00$

Campbell's Early ........... $2.75 \quad 25.00$

Clinton ................. $2.75 \quad 25.00$

Ives $\ldots \ldots \ldots \ldots \ldots \ldots \ldots . \ldots 225 \quad 20.00$

Isabella $\ldots . \ldots \ldots \ldots . \ldots . .2 .25 \quad 20.00$

Moore's Early ............. $2.25 \quad 20.00$

Worden ................. $2.25 \quad 20.00$

Herbert ................ $2.50 \quad 22.00$

-2 Yr. No. 1 -

Concord .................\$2.50 $\$ 20.00$

Champion ................. $3.00 \quad 25.00$

Campbell's Early ........... $3.50 \quad 30.00$

Ives ...................... $3.00 \quad 25.00$

Clinton ................. $3.00 \quad 25.00$

Isabella ................ $3.00 \quad 25.00$

Moore's Early ............ $3.50 \quad 30.00$

Worden .................. $3.50 \quad 30.00$

Herbert ................ 3.5030 .00

(FOR 3 YR., NO. 1, ADD $\$ 3.00$ PER 1000)

(Red Varieties)

-1 Yr. No. 1 and 2 Yr. No. 2-

Agawam ................\$6.00 $\$ 50.00$

Catawba ................6. $6.00 \quad 50.00$

Salem ...................6. $6.00 \quad 50.00$

Diamond .................6. $6.00 \quad 50.00$

Woodruff $\ldots \ldots \ldots \ldots \ldots .6 .600 \quad 50.00$

Brighton ................. $7.00 \quad 6000$

Wyoming Red ............. $7.00 \quad 60.00$

Green Mountain ...........6 $6.00 \quad 50.00$

Niagara ............... $4.00 \quad 35.00$

Delaware .............. $6.00 \quad 50.00$

-2 Yr. No. 1-

Agawam

1001000

Catawba
Salem .................. $7.00 \quad 60.00$

Diamond $\ldots \ldots \ldots \ldots \ldots \ldots \ldots 7.00 \quad 60.00$

Woodruff .............. $7.00 \quad 60.00$

Brighton .............. $8.00 \quad 70.00$

Wyoming Red .......... 8.0070 .00

Niagara ............... $5.00 \quad 4500$

Delaware .............. $7.00 \quad 6000$

-1 Yr. No. 2-

$100 \quad 1000$

Concord ...............\$1.50 \$12.00

Champion ............... $1.50 \quad 12.00$

Campbell's Early ........... 2.00 15.00

Clinton ................. $2.00 \quad 15.00$

Ives $\ldots \ldots \ldots \ldots \ldots \ldots \ldots \ldots 1.50 \quad 12.00$

Isabella $\ldots \ldots \ldots \ldots \ldots \ldots \ldots \ldots . \ldots 1.50 \quad 12.00$

Moore's Early ............. 2.00 15.00

Worden ................. $2.00 \quad 15.00$

Niagara ................. 2.00 15.00

Catawba ............... 2.50 20.00

Agawam ............... $2.50 \quad 20.00$

Diamond ................ $2.50 \quad 20.00$

—No. 3 for Lining Out-

1000

Concord ....................\$ 8.00

Champion .................. 8.00

Campbell's Early ............... 1000

Isabella .................. 10.00

Moore's Early ................... 10.00

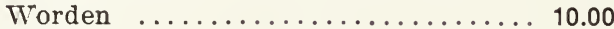

Niagara .................... 12.00

Catawba .................... 15.00

Agawam ............................. 15.00

Moore Diamond ................ 15.00

\section{Grape Cuttings, 8 to 12 inches}

Concord .................. \$1.40

Champion .......................... 1.75

Ives $\ldots \ldots \ldots \ldots \ldots \ldots \ldots \ldots \ldots \ldots . . \ldots \ldots$

Moore's Early ................. 2.25

Worden ...................... 2.50

$\$ 60.00$ Isabella ..................... 2.50

$7.00 \quad 60.00$ Campbell's Early ............... 2.50 


\section{UR R A N T S}

100

London Market, 1 yr. No. $1 \ldots \$ 3.50$

London Market, 2 yr. No. $1 \ldots 4.50$

Wilder, 1 yr. No. $1 \ldots \ldots \ldots . .4 .00$

Wilder, 2 yr. No. $1 \ldots \ldots . \ldots .4 .50$

Fay's Prolific, 1 yr. No. 1 .... 4.00

Fay's Prolific, 2 yr. No. 1 ... 5.00

Cherry, 1 yr. No. 1 ....... 4.50

Red Cross, 1 yr. No. $1 \ldots \ldots .400$

Perfection, 1 yr. No. $1 \ldots \ldots . .60$

55.00

-1 Yr. No. 2-

London Market ............\$1200

Wilder .................. 13.00

Fay's Prolific ................. 13.00

Cherry ................... 13.00

Red Cross .................. 13.00

Perfection .................. 15.00

\section{Currant Cuttings, 7 to 9 inches}

1000

London Market ..............\$3.00

Wilder .................... 4.00

Fay's Prolific and Cherry ......... 4.25

Red Cross .................. 4.00

Perfection ................ 6.00

\section{G O O SEB ER R I ES}

100

Houghton, 1 yr. No. $1 \ldots \ldots \ldots 4.00$

Houghton, 2 yr. No. $1 \ldots \ldots . \ldots 60$

Downing, 1 yr. No. $1 \ldots \ldots$. . 4.50

Downing, 2 yr. No. $1 \ldots \ldots . . .700$

Josselyn, 1 yr. No. $1 \ldots \ldots . . .7 .00$

Josselyn, 2 yr. No. 1 . . . . . . 9.00

Smith Improved, 1 yr. No. 1 . . 4.50

Smith Improved, 2 yr. No. 1 . . 6.00

\section{R E D R A P B E R R I S} (No. I Suckers)

\begin{tabular}{|c|c|}
\hline & 100 \\
\hline Latham &.$\$ 2.50$ \\
\hline & . 2.00 \\
\hline Victory . & 1.50 \\
\hline Cuthbert & $\ldots \ldots \ldots \ldots \ldots \ldots \ldots, 1.40$ \\
\hline $\begin{array}{l}\text { St. Regis } \\
\text { King }\end{array}$ & $\begin{array}{ll}1.50 \\
125\end{array}$ \\
\hline
\end{tabular}

1001) $\$ 2000$

15.00

1300

12.00

13.00

10.00

\section{Transplants No. 1}

Latham ............... \$3.00

Ohta ................. 225

Victory ................. 225

Cuthbert ..............200

St. Regis ............... 2.00

King $\ldots \ldots \ldots \ldots \ldots \ldots \ldots 2.00$
B L A CK RASPBERRIES (No. 1 Tips)

$\begin{array}{rrr}100 & 1000\end{array}$

Cumberland $\ldots \ldots \ldots \ldots \ldots \ldots . \$ 1.40 \quad \$ 12.00$

Plum Farmer ............. $1.40 \quad 12.00$

Gregg ................... $1.40 \quad 12.00$

Kansas .................. $1.40 \quad 12.00$

Columbian Purple ......... $3.00 \quad 25.00$

\section{R H U B A R B}

1000

Myatt's Linneaus, 1 yr. No. 1 .....\$14.00

Myatt's Linneaus, 2 yr. No. 1 ..... 20.00

Mammoth, 1 yr. No. 1 ............ 14.00

Mammoth, 2 yr. No. $1 \ldots \ldots \ldots \ldots .20 .00$

\section{A S P A A G US ROOTS}

1000

Conover and Palmetto, 1 yr. No. $1 \ldots \$ 5.00$

Martha Washington, 1 yr. No. $1 \ldots . .66 .00$

Barr's Mammoth, 1 yr. No. 1 ....... 5.00

Palmetto, 2 yr. No. 1 ........... 9.00

Martha Washington, 2 yr. No. $1 \ldots \ldots .00$

\section{B L A C K B E R R I E S} (No. 1 Suckers)

\begin{tabular}{|c|c|c|}
\hline & 100 & 1000 \\
\hline Eldorado &.$\$ 1.40$ & $\$ 12.00$ \\
\hline Blowers & . 1.40 & 12.00 \\
\hline Mersereau .. & 1.40 & 12.00 \\
\hline Wilson $\ldots \ldots \ldots$ & 1.40 & 12.00 \\
\hline Early Harvest & . 1.40 & 12.00 \\
\hline Lawson ...... & . 1.40 & 12.00 \\
\hline Lucretia Dewb & & 12.00 \\
\hline
\end{tabular}

\section{No. 1 Root Cuttings}

$\begin{array}{rrr}100 & 1000\end{array}$

Eldorado ................\$1.75 $\$ 15.00$

Blowers ................. $1.75 \quad 15.00$

Mersereau ...............2. 2.00 16.00

Lucretia Dewberry .......... $2.00 \quad 16.00$

\section{No. 2 Root Cuttings}

Eldorado, Blowers ..............\$8.00

Mersereau .................. 8.00

Lucretia Dewberry ............ 8.00

\section{4 to 5 -inch Roots}

Eldorado, Mersereau .............\$3.25

$\$ 17.00$ Blowers, Dewberry ............ 3.25 


\section{-STRAWBERRIES-}

1000

Senator Dunlap

$\$ 3.00$

Brandy Wine

4.50

Dr. Burrill

Cooper

500

Gibson

3.50

Premier

4.50

Warfield

3.50

Heritage

Wm. Belt

3.50

Minnehaha

Eaton

3.50

4.50

Pride of Michigan

4.00

Bun's Special

4.00

Samples

4.00

Gandy

4.00

Everbearing

Ároma

4.00

Progressive

Glenn Mary

4.25

Champion

6.00

Glenn Mary

Mastodon

13.00

Your Correspondence Solicited,

\section{J. Rambo's Wholesale Nurseries} BRIDGMAN, MICHIGAN 